\title{
Preparedness planning for pandemic influenza among large US maternity hospitals
}

\author{
RH Beigi ${ }^{1}$, G Davis ${ }^{2}$, J Hodges ${ }^{2}$, and A Akers ${ }^{1}$ \\ ${ }^{1}$ Department of Obstetrics, Gynecology and Reproductive Sciences, Magee-Womens Hospital of the University of Pittsburgh Medical Center, \\ Pittsburgh, PA, USA; and ${ }^{2}$ Magee-Womens Hospital of the University of Pittsburgh Medical Center, Pittsburgh, PA, USA
}

Correspondence

RH Beigi, Department of

Obstetrics, Gynecology and

Reproductive Sciences, Magee-

Womens Hospital of the University

of Pittsburgh Medical Center,

300 Halket Street, Pittsburgh,

PA 15213, USA

E-mail: rbeigi@mail.magee.edu

Received 24 October 2008

Revised 17 December 2008

Accepted 30 December 2008
The objective of this investigation was to determine the state of pandemic influenza preparedness and to delineate commonly reported challenges among a sample of larger US national maternity hospitals. This was done given the recent emphasis on hospital disaster planning and the disproportionate morbidity and mortality that pregnant women have suffered in previous influenza pandemics. An internet-based survey was sent to all 12 members of the Council of Women's and Infants' Specialty Hospitals. Questions addressed hospital demographics and overall pandemic preparedness planning, including presence of a pandemic planning committee and the existence of written plans addressing communications, surge capacity, degradation of services, and advance supply planning. Nine of $12(75 \%)$ hospitals responded. All had active pandemic planning committees with identified leadership. The majority (78\%) had written formal plans regarding back-up communications, surge/overflow capacity, and degradation of services. However, fewer (44\%) reported having written plans in place regarding supply-line/stockpiling of resources. The most common challenges noted were staff and supply coordination, ethical distribution of limited medical resources, and coordination with government agencies. In conclusion, the majority of the Council of Women's and Infants' Specialty Hospitals maternity hospitals have preliminary infrastructure for pandemic influenza planning, but many challenges exist to optimize maternal and fetal outcomes during the next influenza pandemic.

\section{Introduction}

Hospital planning for disaster preparedness has become a growing area of domestic attention given the large numbers of natural and man-made disasters that have occurred since September 11 2001. One of the major focus areas has centered on hospital preparedness for pandemic influenza. Pandemic planning is of major importance to hospitals providing maternity and newborn care given the disproportionate morbidity, mortality, and preterm birth rates recorded among pregnant women with influenza pneumonia during the previous 20th century influenza pandemics. ${ }^{1-5}$ Historic records from the 1918 influenza pandemic suggest that the maternal mortality rate was two to three times higher than in the general population. The pregnancy loss rate, both spontaneous abortions and preterm deliveries, was in excess of $50 \%$ for symptomatic pregnant women. ${ }^{2,3}$ Thus, active planning for a potentially disastrous influenza pandemic is a critically important endeavor for healthcare professionals and healthcare administrators, particularly those caring for pregnant women and newborns.
Although it is difficult to predict when an influenza pandemic will occur, large-scale outbreaks have been reported approximately every 10-40 years. The emergence of H5N1 avian influenza in 1997, in addition to the fact that nearly 40 years have passed since the last influenza pandemic, makes the threat of a severe influenza pandemic a realistic possibility in the near future. ${ }^{6}$ To date, there have been 387 confirmed human cases of $\mathrm{H} 5 \mathrm{~N} 1$ influenza reported to the World Health Organization (WHO), and of those 245 have died (63.3\% mortality). ${ }^{7}$ Importantly, the majority of those infected have been young healthy individuals, exactly matching the demographic profile of reproductive age women primarily cared for in hospitals providing maternity care. ${ }^{8}$

The Council of Women's and Infants' Specialty Hospitals (CWISH) is a membership organization of maternity hospitals founded in 1991, with the goal of sharing information and operations data to improve maternity and newborn care in the USA. Currently, there are 12 member hospitals representing all major US geographic regions that together

This is an Open Access article distributed under the terms of the Creative Commons Attribution licence which permits unrestricted use, distribution, and open - access reproduction in any medium, provided the original work is properly cited. 
account for $>110,000$ deliveries each year. The member hospitals are located in diverse settings including urban, suburban, and rural locations. Thus, CWISH represents a consortium of geographically and functionally diverse large maternity hospitals that currently deal with large-scale operations for providing maternity and newborn care. These hospitals can offer important and diverse insights about how to plan for and manage disaster efforts.

The primary goal of this study was to investigate the current state of pandemic influenza planning for obstetrical patients among CWISH hospitals and to identify major challenges to planning efforts. Although any institutional plan to prepare for an influenza pandemic would include mobilization of resources and planning for newborn care as well as hospital-wide preparedness, our focus in this study was in specifically assessing obstetric care planning issues. Our broader goals were to use this data to (1) facilitate information exchange regarding pandemic influenza preparedness between CWISH hospitals, (2) provide US national-level data regarding planning efforts, and (3) stimulate ongoing pandemic planning efforts at all hospitals providing maternity and/or neonatal care. This investigation can provide important data for other US hospitals providing maternity care that are interested in or who are currently undertaking pandemic influenza planning efforts. In addition, the delineation of major challenges encountered among large maternity hospitals is critical for improving national perinatal preparedness and optimizing the care of pregnant women, their unborn fetuses, and neonates during the next influenza pandemic.

\section{Methods}

\section{Study design}

This investigation involved an internet-based survey of all 12 hospitals comprising the CWISH consortium conducted in the spring and summer of 2007.

\section{Questionnaire}

The questionnaire contained 11 items that assessed each hospital's demographic characteristics, pandemic preparedness as well as perceived and real challenges to pandemic planning. The questionnaire items were developed by the first author in consultation with local and national experts in pandemic influenza preparedness. The published guidelines on hospital preparedness were also consulted to assure that the major focus areas were relevant and consistent with national consensus on this topic. ${ }^{9-11}$ The final questionnaire instrument was reviewed and refined by all the authors including a local expert in survey design.

\section{Demographics}

One hospital demographic item was included that assessed the number of annual obstetric deliveries. There were four response options selected based on the demographics of CWISH-member hospitals: (1) <5000, (2) 5000-7499, (3) 7500-9999, and (4) >10,000 deliveries per year.
Pandemic preparedness

Nine items assessed the state of each hospital's pandemic preparedness. These items covered five content domains, including (1) whether a facility had a formal pandemic planning committee (PPC), (2) the presence and qualifications of their PPC administrative leader, (3) the status of planning related to four nationally identified key areas for disaster planning, (4) the presence or absence of human resource management planning for disaster relief efforts, and (5) the existence of ethical and logistical planning for obstetric care in the event of a disaster.

\section{Committee leadership}

PPC leadership was assessed with two items. The first asked whether a facility's hospital administration had identified an individual to lead the hospital's pandemic planning efforts. The second asked respondents to describe the leader's primary job description using the following response options: physician, nurse, administrative, or other. Participants responding 'other' were asked to write in their response.

\section{Pandemic planning}

The status of pandemic planning was assessed with an item that asked respondents to assess their facility's formal plans regarding each of the four nationally identified key areas for pandemic planning: (1) back-up communication planning, (2) surge capacity planning (that is, where to overflow excess patients), (3) degradation of services planning, and (4) supply-lines investigation and stockpiling of essential equipment, medications, and other medical supplies. The response options were 'item not yet considered', 'item discussed', 'plan being developed', and 'formal plan in place'.

\section{Human resource management planning}

Human resource management planning was assessed with two items. Facilities were asked whether they planned to provide medical care to and/or day-care services for affected staff members. Two items then asked whether facilities had considered two issues specific to the ethics and logistical planning for obstetric care during a pandemic: ethical issues related to the rationing of care for pregnant women in times of limited resources and logistical planning for where to overflow labor and delivery services once their hospital surpassed surge capacity. The consideration of overflow sites was added to address not only the potential need for overflow services but also to assess the potential for separation between infected and noninfected pregnant women. Finally, two open-ended questions assessed respondents' perceived and real challenges to pandemic planning.

\section{Data collection and analysis}

The contact person listed on the CWISH registry was contacted through electronic mail and asked to complete the internet-based survey. Two subsequent monthly 
Table 1 Nationally recommended focus areas for hospital pandemic planning

\begin{tabular}{lcc}
\hline Key focus area & $\begin{array}{c}\text { Currently formulating } \\
\text { written plan N (\%) }\end{array}$ & $\begin{array}{l}\text { Have formal written } \\
\text { plan in place N (\%) }\end{array}$ \\
\hline $\begin{array}{l}\text { Back-up communications } \\
\text { planning }\end{array}$ & $2(22.2)$ & $7(77.8)$ \\
$\begin{array}{l}\text { Surge capacity planning } \\
\text { Degradation of services } \\
\text { planning }\end{array}$ & $2(22.2)$ & $7(77.8)$ \\
$\begin{array}{l}\text { Stockpiling and supply-line } \\
\text { planning }\end{array}$ & $2(22.2)$ & $7(77.8)$ \\
\hline
\end{tabular}

${ }^{a}$ Addresses the ability of a facility to increase the numbers of patients simultaneously receiving inpatient services at any one time.

${ }^{\mathrm{b}}$ Addresses the practice of a hospital temporarily suspending some of the nonessential services it offers in order to increase the proportion of critical services (that is, temporary cancellation of elective surgical procedures).

reminder notices were sent to hospitals that did not respond after the first contact. Descriptive statistics were used to describe the frequency of responses for each quantitative item. Qualitative responses were grouped for each question and examined for each individual facility and across facilities to identify common and unique themes.

\section{Results}

Nine of the participating $12(75.0 \%)$ CWISH hospitals responded to the survey. All of the respondent hospitals perform $>5000$ deliveries per year. Six (67.0\%) perform $>7500$ and two (22\%) perform $>10,000$ deliveries per year. Seven of the nine (78\%) responding hospitals are free-standing maternity hospitals, and the other two are large maternity operations functioning within larger full-service hospital facilities. All of the CWISH hospitals have self-contained critical care and obstetric anesthesia services.

All nine (100\%) reported that they had an existing PPC in place with identified leadership on the committee. The individuals with leadership roles on the committee included obstetric and nonobstetric physicians, maternity and medical/surgical nurses, hospital administrators, infection control practitioners, risk managers, and employee health personnel.

Facilities' formal plans based on the four key areas for hospital pandemic planning are shown in Table 1 . Hospitals were then asked whether they had begun to address the ethical considerations such as distribution of limited resources or rationing of supply and care during an impending influenza pandemic. Fifty-six percent answered yes, 22\% stated no, and the other $22 \%$ were unsure. No hospital responded that they had a formal plan already in place to address these ethical considerations.

Regarding human resource management during a pandemic, all facilities planned to provide care to their own employees (both female and male) despite the potential for significant shortages of both personnel and supplies. Five (56\%) facilities also planned to provide augmented day-care
Table 2 Common challenges and areas of concern listed by CWISH hospitals

\begin{tabular}{ll}
\hline Common challenges & Staff coordination \\
& Supply coordination \\
& Hospital patient flow and infection control \\
& Medication distribution \\
& Ethics and rationing of care considerations \\
& Coordination with state health agencies \\
Other concerning areas & Employee screening into workplace \\
& Visitation policies for laboring mothers \\
& Food supplies and storage \\
& Mortuary capacity \\
& Employee child-care \\
Communication with government
\end{tabular}

Abbreviation: CWISH, Council of Women's and Infants' Specialty Hospitals.

services to the children of their employees during an influenza pandemic to increase the ability of workers to continue to come to work. Six (67\%) of the facilities had identified an overflow site in the hospital for surge labor and delivery services. Locations for labor and delivery overflow included adult same day surgery units, adult intensive care units, and hospital surgical suites.

The common challenges expressed by participant hospitals are displayed in Table 2 . Of note, the most commonly cited challenge areas among all the respondent hospitals were staff and supply coordination, ethical distribution of limited medical resources, and hospital coordination with government agencies.

\section{Discussion}

The results of this survey of a sample of large US maternity hospitals show that the majority of responders have made significant, preliminary strides in terms of developing basic infrastructure and planning for obstetric care in preparation for the next influenza pandemic. This is encouraging given the recorded disproportionate morbidity, mortality, and fetal wastage demonstrated in the 20th century influenza pandemics among pregnant women with pneumonia. Fortunately, the majority of respondents reported having formal written plans addressing some of the major focus areas for hospital pandemic planning recognized nationally; ${ }^{9-11}$ for those not yet with formal documents, planning was already in progress.

For any hospital providing maternity care, the items queried herein represent an excellent starting point for planning efforts. The first step would be the formation of a multidisciplinary PPC with identified leadership that can begin to address these nationally emphasized focus areas (Table 1) in a facility-specific manner. Although it is not abundantly clear how well modern medicine will be able to prevent devastation during such a large-scale infectious disease disaster, it is widely recognized that advance planning holds promise to temper the extent of devastation. This becomes especially important for vulnerable populations that are often left out of national level consideration, such as pregnant women, fetuses, and neonates. 
One of the strengths of our approach was that we sought to describe the status of pandemic planning among large maternity care hospitals using national guidelines for disaster planning. We also went one step further and asked hospitals to identify other major challenges that they have faced in their pandemic planning efforts that might not be adequately captured by existing national disaster planning guidelines. The key challenges listed include staff and supply coordination, the ethical distribution of limited medical resources, and hospital coordination with federal agencies. Identification of these potentially relevant challenge areas may serve as target areas for national planning efforts.

Delineating an ethical framework for prioritizing and rationing medical services during a pandemic was a major challenge commonly articulated by the hospitals surveyed. There is some literature that addresses ethical issues in rationing for the general medical population, however, none addresses rationing and/or prioritization for pregnant women and newborns specifically. ${ }^{12-14}$ This void in guidance is important given the important differences between general adult medical patients and obstetric or neonatal patient populations. Unlike most general medical patients, obstetric and neonatal patients are likely to have an increased susceptibility to the infectious agent. Despite this heightened susceptibility, less robust data exist on dosing for both pregnant women and neonates with antimicrobials to combat these infectious diseases. These and many other factors make pregnant women and neonates special populations justifying preemptive ethical consideration.

National literature regarding disaster planning suggests using a severity index to triage patients presenting to the hospital to determine who may be good candidates to receive the limited resources. These severity indices take the presence of significant comorbidities into consideration and/ or seek to identify patients whose situations are potentially clinically futile. Although this approach will likely be very effective for older patients with significant comorbidities, it is doubtful that a large percentage of maternity patients will have significant comorbidities. Therefore, many will not 'screen out' of the first round of resource triage, resulting in a significant percentage of maternity patients still requiring decisions on how to prioritize the limited resources.

An additional commonly mentioned area of concern was the coordination of hospitals with local, state, and federal government public health agencies. It is unclear for many institutions (especially private hospitals) how exactly they might interface with public health agencies. Despite the lack of clarity in these relationships, public health agencies will likely play a critical role in the provision of information, medications, and vaccine dissemination as they will be supplied by the federal strategic national stockpile and may have the most accurate outbreak information as the pandemic unfolds.

Several limitations to the current investigation are worth noting. Primarily, we surveyed a small sample of the nation's largest maternity hospitals. As such, the data may not truly represent the actual state of pandemic planning nationally. Indeed, it is likely that the respondent hospitals are further along in planning for maternity and neonatal care than most hospitals that provide more diverse patient care. Moreover, the actual written plans of each hospital were not reviewed by our institution, but rather reports in direct response to our targeted queries by the respective hospitals were reviewed. Although this method may not accurately depict the exact nature and status of the plan, these findings do indicate that influenza preparedness planning is taking place nationally. In addition, this investigation can serve as a model for other hospitals providing maternity care services regarding how to proceed with planning efforts for an impending disaster, such as pandemic influenza. Another consideration is that a quarter of CWISH hospitals $(n=3)$ did not respond to this survey. It is not clear why nonresponders chose not to participate or how they differ in their disaster planning efforts from institutions that did participate. However, the three nonrespondent hospitals do not differ substantially in their services offered or the populations they serve when compared with the nine responders. Lastly, we focused on pandemic planning related to obstetric patients but did not assess newborn or hospital-wide planning efforts. It is recognized that services for the newborn will also likely be critically overstretched in the face of the next influenza pandemic. Future efforts should consider assessing these two services together to fully address institutional preparedness for a future influenza pandemic.

In conclusion, these data suggest that some of the nation's larger maternity hospitals have significant planning underway for an influenza pandemic. However, many challenges remain that need to be addressed at the local, state, and federal level, if we are to improve on our efforts to optimize outcomes for pregnant women and newborns during the next influenza pandemic. It is hoped that this investigation will stimulate ongoing planning efforts for all hospitals providing maternity care in preparation for this apparent inevitable infectious disease outbreak.

\section{Acknowledgements}

This work was previously presented in oral abstract format at the American College of Obstetricians and Gynecologists (ACOG) Annual Clinical Meeting May 2008 in New Orleans, LA, USA.

\section{Author contributions}

All authors contributed equally to the development, intellectual concepts, and writing of this paper.

\section{Conflict of interest}

The authors have no conflict of interest.

\section{Ethical approval}

Data for the article were initially part of a quality assurance initiative that did not include human subject risk. Upon 
completion of the survey, the authors realised that the uniqueness of the data warranted publication. The collection of survey information was not originally planned as a research protocol. IRB approval was considered, but not sought, as the research did not involve any human subject risk.

\section{Funding}

This study was supported by departmental funding.

Provenance and peer review

Not commissioned; externally peer-reviewed.

\section{References}

1 CDC. Prevention and control of influenza: recommendations of the Advisory Committee on Immunization Practices. MMWR 2006;55(Early Release):1-41.

2 Woolston WJ, Conley DO. Epidemic pneumonia (Spanish Influenza) in pregnancy: effect in one hundred and one cases. IAMA 1918;71:1898-9.

3 Harris JW. Influenza occurring in pregnant women: a statistical study of thirteen hundred and fifty cases. JAMA 1919;72:978-80.

4 Greenberg M, Jacobziner H, Pakter J, Weisl BAG. Maternal mortality in the epidemic of Asian influenza, New York City, 1957. Am J Obstet Gynecol 1958;76:897-902.
5 Freeman DW, Barno A. Deaths from Asian influenza associated with pregnancy. Am J Obstet Gynecol 1959;78:1172-5.

6 Webster RG, Govorkova EA. H5N1 - Continuing evolution and spread. N Engl J Med 2006;355:2174-7.

7 World Health Organization. Cumulative number of confirmed human cases of avian influenza $\mathrm{A} /(\mathrm{H} 5 \mathrm{~N} 1)$ reported to WHO. Available at: http://www.who.int/csr/disease/avian_influenza. Retrieved September 17, 2008.

8 Beigel JH, Farrar J, Han AM, Hayden FG, Hyer R, de Jong MD, et al. Avian influenza A (H5N1) infection in humans. N Engl J Med 2005;353:1374-85.

9 Toner E, Waldhorn R, Maldin B, Borio L, Nuzzo JB, Lam C, et al. Hospital preparedness for pandemic influenza. Biosecur Bioterror 2006;4:207-17.

10 Toner E, Waldhorn R. What should hospitals do to prepare for an influenza pandemic. Biosecur Bioterror 2006;4:397-402.

11 Maldin B, Lam C, Franco C, Press D, Waldhorn R, Toner E, et al. Regional approaches to hospital preparedness. Biosecur Bioterror 2007;5:43-53.

12 Christian MD, Hawryluck L, Wax RS, Cook T, Lazar NM, Herridge MS, et al. Development of a triage protocol for critical care during an influenza pandemic. CMAJ 2006;175:1377-81.

13 Hick JL, Rubinson L, O'Laughlin DT, Farmer JC. Clinical review: allocating ventilators during large-scale disasters-problems, planning, and process. Crit Care 2007;11:1-9.

14 Hick JL, O'Laughlin DT. Concept of operations for triage of mechanical ventilation in an epidemic. Acad Emerg Med 2006;13:223-9. 